Д.Е. Ситніков, П.Е. Ситнікова, С.В. Тітов, О.В. Тітова

Харківський національний університет радіоелектроніки, Харків

\title{
ФІЛЬТРАЦІЯ РЕЗУЛЬТУЮЧОГО НАБОРУ АСОЦІАТИВНИХ ПРАВИЛ 3 ТОЧКИ ЗОРУ ОЦІНКИ ЦІКАВОСТІ
}

\begin{abstract}
Пропонується метод фільтращії набору асоичітивних правил, отриманих у результаті пошуку логічних залежностей. Кількість знайдених асоціативних правил за умови встановлених рівнів підтримки та довіри може бути досить великою й потребує скорочення. Метод дозволяє працювати з так званими "цікавими" правилами, які мають такі рівні підтримки та довіри, які значно відрізняються від очікуваних. Очікувані параметри розраховуються виходячи з припущення про незалежність ознак, щчо входять до лівої частини правила. Показано, як змінюються рівні підтримки та довіри "цікавих" асоціативних правил за умови залежності ознак в даних, які аналізуються.
\end{abstract}

Ключові слова: асочіативні правила, міри оцінки асочіативної залежності, підтримка, довіра, рівень поліпшення, цікаві асоціативні правила.

\section{Вступ}

Постановка проблеми. До основних завдань вилучення знань 3 даних у вигляді знаходження регулярностей у великих масивах інформації, (Data Mining) відносяться:

1) побудова класифікатора;

2) кластеризація;

3) генерація правил асоціації;

4) відновлення моделей залежності.

Для вирішення реальної практичної задачі, як правило, потрібне застосування декількох підходів і методів.

Пошук асоціативних правил полягає у виведенні правил імплікативного виду, де кожне правило відображає набір взаємно супутніх подій. Спочатку це завдання виникло як прикладне в сфері маркетингу (так званий "кошик покупок"). Виявлялися товари, які покупці намагались купувати разом. Згодом завдання пошуку асоціативних правил було сформульовано в більш загальному вигляді як завдання знаходження логічних закономірностей в даних. Ці правила можуть використовуватися для аналізу даних i прийняття рішень, а також після відповідної оцінки служити вихідним матеріалом для побудови баз знань.

Для генерації асоціативних правил існує ряд алгоритмів, найбільш відомим з яких є алгоритм Apriori [1-2] та його модифікації (AprioriTid, AprioriHybrid та ін.). Незважаючи та те, що ці алгоритми існують досить давно (Apriori був запропонований у 1994 р.), на ринку програмного забезпечення слабо представлені їx реалізації для вільного та комерційного використання. Одним з продуктів, які дозволяють знаходити логічні залежності у вигляді правил $X \rightarrow Y$, є системa WizWhy підприємства WizSoft [3]. Для генерації асоціативних правил встановлюється ряд параметрів, необхідних для отримання результуючого набору логічних залежностей. Найбільш відомими серед них $\epsilon$ рівень підтримки Support (Supp) (визначає кількість об'єктів (записів в БД), для яких виконується це правило) та рівень довіри Confidence (Conf) (відносна частота виконання правила на досліджуваному наборі даних).

Однією з проблем під час пошуку асоціативних правил в даних $\epsilon$ той факт, що число залежностей може бути досить великим. Таким чином, результуючий набір потребує фільтрації результатів 3 метою вилучення 3 нього неінформативних, дублюючих правил. Безумовно, ця задача не може бути повністю вирішена без залучення людини-аналітика, але окремі завдання із скорочення кількості отриманих асоціативних правил підлягають автоматизації.

Аналіз останніх досліджень і публікацій. Для скорочення результуючого набору асоціативних правил застосовують різні підходи. По-перше, встановлення ряду обмежень на отримані логічні залежності - а саме визначення необхідних рівнів підтримки та довіри [1-2].

Однак цих мір також виявляється недостатньо. У роботах [4-6] пропонуються додаткові міри для оцінки асоціацій, а саме: обгрунтованість (Validity), впевненість (Conviction), рівень поліпшення (Improvement/Lift) та ін.

Наприклад в WizWhy [3] обмеженням є довжина правила. Тобто максимальна довжина комбінації в правилі "якщо ... то ..." в системі WizWhy дорівнює 6 та 3 самого початку роботи алгоритму проводиться евристичний пошук простих логічних подій, на яких потім будується весь подальший аналіз. 
У роботах [7-8] описано метод оцінки асоціативних правил з точки зору теорії інформації, який дозволяє виключити з результуючого набору правил неінформативні логічні залежності. Цей метод дозволяє знаходити інтегральну міру асоціації - повну взаємну інформацію, враховуючи такі параметри асоціативного правила, як рівень підтримки, рівень довіри та рівень поліпшення.

Мета статті - опис методу скорочення результуючого набору логічних залежностей для "цікавих асоціативних правил", тобто для правил параметри яких (Supp, Conf) істотно відрізняються від очікуваних.

\section{Виклад основного матеріалу}

Розглянемо так звані "цікаві" правила або "цікаві феномени" за визначенням системи WizWhy.

Під "цікавим" правилом розуміється правило, параметри (Supp, Conf) якого істотно відрізняються від очікуваних.

Наприклад:

- нехай $A$ - деякий симптом, який 3 ймовірністю $60 \%$ говорить про наявність у пацієнта хвороби $D$;

- нехай $B$ - деякий симптом, який з ймовірністю $60 \%$ говорить про наявність у пацієнта хвороби $D$;

- якщо у пацієнта присутні обидва симптоми, то ймовірність хвороби $D$ виявляється $10 \%$.

Під ймовірністю у даному випадку розуміється рівень довіри (Conf - "Confidence") асоціативного правила.

Розглянемо, яким чином може бути розрахований очікуваний рівень підтримки для правила $A B \rightarrow D$.

$\operatorname{Supp}(A \rightarrow D)$ дорівнюе ймовірності (відносній частоті, але в даному контексті будемо використовувати поняття ймовірності) того, що запис містить i ознаку $A$, і ознаку $D$, тобто

$$
\operatorname{Supp}(A \rightarrow D)=P(A D)=\frac{N_{A D}}{N_{D S}},
$$

де $N_{A D}$ - множина записів, які містять ознаку $A$ i ознаку $D$;

$N_{D S}$ - множина записів, що становлять Data Set.

Відповідно, $\operatorname{Supp}(B \rightarrow D)=P(B D)$.

Якщо $A$ i $D$ - незалежні, то $\operatorname{Supp}(A \rightarrow D)=\operatorname{Supp}(A) \cdot \operatorname{Supp}(D)$. Безумовно, такі правила не представляють інтересу з точки зору пошуку залежностей у даних. Відзначимо, що такий параметр як Improvement (в деяких джерелах його називають Lift) також $є$ індикатором "незалежності" правої і лівої частин асоціативного правила (в цьому випадку він дорівнює 1).

$$
\operatorname{Imp}(A \rightarrow D)=\frac{P(A D)}{P(A) P(D)} .
$$

Звернемося тепер до "цікавих" правил з точки зору розробників системи WizWhy. У таких правилах задіяні як мінімум дві ознаки, (позначимо їх $A$ i $B)$ i "залежна змінна" (позначимо іiі через $D$ ), яка представляє праву частину правила $A B \rightarrow D$.

Розрахуємо очікуваний рівень підтримки $\operatorname{Supp}_{\exp }(A B \rightarrow D)$ для правила, беручи до уваги, що нам відомі рівні підтримки правил $A \rightarrow D$ та $B \rightarrow D$.

Для цього скористаємося відомою формулою теорії ймовірностей для суми трьох сумісних подій:

$$
\begin{aligned}
& P(A+B+D)=P(A)+P(B)+P(D)- \\
& -P(A B)-P(A D)-P(B D)+P(A B D)
\end{aligned}
$$

звідси

$$
\begin{aligned}
& P_{\exp }(A B D)=\operatorname{Supp}_{\exp }(A B \rightarrow D)= \\
& =P(A+B+D)-P(A)-P(B)-P(D)+ \\
& +P_{\exp }(A B)+P(A D)+P(B D) .
\end{aligned}
$$

У формулі (1):

$P(A+B+D)=\operatorname{Supp}(A+B+D)-$ відносна кількість записів в Data Set, що містять ознаки $A$ або $B$ або $D$;

$P(A)$ - відносна кількість записів в Data Set, що містять ознаки $A(P(B)$ і $P(D)$ аналогічно);

$$
P(A D)=\operatorname{Supp}(A \rightarrow D) \text { - відносна кількість за- }
$$
писів в Data Set, що містять ознаки $A$ і $D(P(A B)$ i $P(B D)$ аналогічно).

Таким чином, знаючи $\operatorname{Supp}(A \rightarrow D)$, $\operatorname{Supp}(B \rightarrow D)$ і припустивши, що $A$ і $B$ - незалежні, тобто $P_{\exp }(A B)=P(A) P(B)=\operatorname{Supp}_{\exp }(A B)$, ми можемо розрахувати очікуваний рівень підтримки правила $A B \rightarrow D$.

Правило $A B \rightarrow D$ є "цікавим", якщо фактичний рівень підтримки істотно відрізняється від очікуваного. Це є показником того, що $P(A B) \neq P(A) P(B)$. Тобто між ознаками $A$ і $B$ існує залежність.

Причому, $\operatorname{Supp}(A B \rightarrow D)<\operatorname{Supp}_{\exp }(A B \rightarrow D)$ означає, що $P_{A}(B)<P(B)$ i $P_{B}(A)<P(A)$.

I навпаки, $\operatorname{Supp}(A B \rightarrow D)>\operatorname{Supp}_{\exp }(A B \rightarrow D)$, означає, що $P_{A}(B)>P(B)$ i $P_{B}(A)>P(A)$.

Очікуваний рівень довіри $\operatorname{Conf}_{\exp }(A B \rightarrow D)$ розраховується наступним чином: 


$$
\operatorname{Conf}_{\exp }(A B \rightarrow D)=\frac{\operatorname{Supp}_{\exp }(A B \rightarrow D)}{\operatorname{Supp}_{\exp }(A B)} .
$$

Проаналізуємо, як зміниться фактичне значення $\operatorname{Conf}(A B \rightarrow D)$ в разі, якщо ознаки $A$ i $B$ залежні.

Якщо

$$
P(A B)<P(A) P(B),
$$

тобто

$\operatorname{Supp}(A B)<\operatorname{Supp}_{\exp }(A B)$, то $\operatorname{Conf}(A B \rightarrow D)$ також зменшиться. Цей висновок можна зробити на підставі того факту, що чисельник і знаменник у формулі (2) зменшуються на одне і теж позитивне число $\Delta=P_{\exp }(A B)-P(A B)$.

Таким чином,

отримаємо: $\operatorname{Conf}(A B \rightarrow D)<$ Conf $_{\text {exp }}(A B \rightarrow D)$.

Якщо $P(A B)>P(A) P(B)$, тобто $\operatorname{Supp}(A B)>\operatorname{Supp}_{\exp }(A B)$, то $\operatorname{Conf}(A B \rightarrow D)$ збільшиться. Таким чином, $\operatorname{Conf}(A B \rightarrow D)>\operatorname{Conf}_{\exp }(A B \rightarrow D)$.

Звідси можна зробити висновок, що "цікаве" правило $A B \rightarrow D$ завжди має відмінні від очікуваних рівень підтримки і рівень довіри, причому, якщо $\operatorname{Supp}(A B \rightarrow D)<\operatorname{Supp}_{\exp }(A B \rightarrow D)$, To

$\operatorname{Conf}(A B \rightarrow D)<\operatorname{Conf}_{\exp }(A B \rightarrow D)$

$$
\text { I навпаки, }
$$

якщо

$\operatorname{Supp}(A B \rightarrow D)>\operatorname{Supp}_{\exp }(A B \rightarrow D)$ TO

$\operatorname{Conf}(A B \rightarrow D)>\operatorname{Conf}_{\exp }(A B \rightarrow D)$.

Для задач аналізу даних це означає, що якщо фактичні рівні підтримки і довіри правила $A B \rightarrow D$ істотно менше очікуваних, (поява ознаки $A$ істотно знижує ймовірність появи ознаки $B$ ), то в результуючому наборі правил повинні бути присутніми правила $A \rightarrow D$ i $B \rightarrow D$.

Якщо ж фактичні рівні підтримки і довіри правила $A B \rightarrow D$ істотно вище очікуваних, (поява ознаки $A$ істотно підвищує ймовірність появи ознаки $B$ ), то в результуючому наборі правил може бути залишено правило $A \rightarrow D$ або $B \rightarrow D$.

Розглянемо тепер поведінку такої характеристики "цікавого" асоціативного правила, як Improvement (Lift).

За визначенням,

$$
\operatorname{Imp}(A B \rightarrow D)=\frac{P(A B D)}{P(A B) P(D)}=\frac{\operatorname{Conf}(A B \rightarrow D)}{\operatorname{Supp}(D)}
$$

Оскільки $\operatorname{Supp}(D)$ для очікуваного і фактичного результату не змінюється, то поведінка такої характеристики як Imp буде залежати від Conf і, відповідно, від Supp.
Отже, правила з характеристиками (Supp, Conf i Imp) менше очікуваних дозволяють стверджувати, що ознаки $A$ і $B$ мають "негативну" залежність і замість правила $A B \rightarrow D$ необхідно розглядати правила $A \rightarrow D$ i $B \rightarrow D$.

Правила 3 характеристиками (Supp, Conf i Imp) більше очікуваних дозволяють стверджувати, що ознаки $A$ і $B$ мають "позитивну" залежність і замість правила $A B \rightarrow D$ необхідно розглядати правила $A \rightarrow D$ або $B \rightarrow D$.

Позначимо різницю очікуваного і фактичного рівнів підтримки як $\Delta_{S}$.

$$
\Delta_{S}=\left|\operatorname{Supp}_{\exp }(A B \rightarrow D)-\operatorname{Supp}(A B \rightarrow D)\right| .
$$

Виходячи $з$ формули (1) можна записати:

$$
\begin{aligned}
& \Delta_{S}=\left|P(A) P(B)-P(A) P_{A}(B)\right|= \\
& =|\operatorname{Supp}(A) \operatorname{Supp}(B)-\operatorname{Supp}(A) \operatorname{Conf}(A \rightarrow B)| .
\end{aligned}
$$

Для простоти опустимо знак модуля, тобто вважатимемо, що $\Delta_{S}>0$.

Тоді

$\Delta_{S}=\operatorname{Supp}(A) \operatorname{Supp}(B)-\operatorname{Supp}(A) \operatorname{Conf}(A \rightarrow B)$.

Звідси:

$$
\operatorname{Conf}(A \rightarrow B)=\frac{\operatorname{Supp}(A) \operatorname{Supp}(B)-\Delta_{S}}{\operatorname{Supp}(A)}
$$

якщо $\Delta_{S}<0$, то

$$
\operatorname{Conf}(A \rightarrow B)=\frac{\Delta_{S}-\operatorname{Supp}(A) \operatorname{Supp}(B)}{\operatorname{Supp}(A)} .
$$

Рівень підтримки правила $A \rightarrow B$ дорівнюватиме:

$$
\operatorname{Supp}(A \rightarrow B)=\left|\operatorname{Supp}(A) \operatorname{Supp}(B)-\Delta_{S}\right| .
$$

Таким чином, знаючи різницю очікуваного i фактичного рівнів підтримки правила $A B \rightarrow D$ (рівень "цікавості"), ми можемо визначити параметри правила $A \rightarrow B$.

Це дозволяє отримати додаткову інформацію під час аналізу цікавих правил (так званих феноменів) в результуючому наборі логічних залежностей.

\section{Висновки}

Запропоновано метод скорочення набору асоціативних правил під час фільтрації результатів пошуку логічних залежностей в досліджуваних наборах даних.

Розглянуто так звані "цікаві" правила, для яких рівень підтримки значно відрізняється від очікуваного. Очікуваний рівень підтримки розраховується 
виходячи 3 припущення про незалежність ознак, які входять до лівої частини правила. Показано, що у випадку, коли рівень підтримки (і як наслідок рівень довіри) менш за очікуваний, то у результуючому наборі повинні бути залишені правила $A \rightarrow D$ і $B \rightarrow D$. Навпаки, якщо коли рівень підтримки і рівень довіри більше за очікуваний, то у результуючому наборі залишається правило $A \rightarrow D$ або $B \rightarrow D$.

Також виходячи 3 даних про фактичну різницю між очікуваними та наявними параметрами правила
$A B \rightarrow D$ можна визначити параметри правила $A \rightarrow B$. Під час роботи з системою WizWhy для генерації асоціативних правил встановлюється залежна змінна, тобто ознака у правій частині правила. За умови знаходження "цікавих" правил, результуючий набір може бути доповнений асоціативним правилом $A \rightarrow B$, що надасть аналітику додаткову інформацію про залежність між ознаками у лівій частині асоціативного правила.

\section{Список літератури}

1. Agrawal R. Fast algorithms for mining association rules / R. Agrawal, R.Srikant // Proceedings of the $20^{\text {th }}$ VLDB Conference Santiago. - Chile, September 1994. - P. 487-499.

2. Agrawal R. Mining generalized association rules / R. Agrawal, R.Srikant // Proceedings of the 21th VLDB Conference Zurich. - Swizerland, September 1995. - P. 407-419.

3. WizWhy user's guide. - URL: https://www.wizsoft.com/n/downloads/User_Manual_WizWhy_4.pdf.

4. A Novel Method of Interestingness Measures for Association Rules Mining Based on Profit / Chunhua Ju, Fuguang Bao, Chonghuan Xu, Xiaokang Fu // Hindawi Publishing Corporation Discrete Dynamics in Nature and Society. - 2015. - Vol. 2015. http://dx.doi.org/10.1155/2015/868634.

5. Victor S.P. Effective measures in Association Rule Mining / S.P. Victor, J.R. Jeba // International Journal of Scientific \& Engineering Research. - 2012. - Vol. 3, Issue 8.

6. Izwan Nizal Mohd. Shaharanee. Interestingness measures for association rules based on statistical validity / Izwan Nizal Mohd. Shaharanee, Fedja Hadzic, Tharam S. Dillon // KnowledgeBased Systems. - 2011. - No. 24. - P. $386-392$. https://doi.org/10.1016/j.knosys.2010.11.005.

7. Dinesh J. Prajapati. Interesting association rule mining with consistent and inconsistent rule detection from big sales data in distributed environment / Dinesh J. Prajapati, Sanjay Garg, N.C. Chauhan // Future Computing and Informatics Journal. 2017. - No. 2. - P. 19-30.

8. Stephane L. Association rule interestingness: measure and statistical validation / L. Stephane, O. Teytaud, E. Prudhomme // Studies in Computational Intelligence (SCI). - 2007. - Vol. 43. - P. 251-275.

9. Aydin T. Modeling interestingness of streaming association rules as a benefit-maximizing classification problem / T. Aydın, HA. Guvenir // Knowledge-based systems. Elsevier. - 2009. - P. 85-99.

10. Ситников Д.Э. Полная взаимная информация как обобщенный показатель качества ассоциативных зависимостей с бинарными признаками [Текст] / Д.Э. Ситников, Е.В. Титова // Системи обробки інформації - 2004. - № 2. C. 20-28.

11. Informativity of Association Rules from the Viewpoint of Information Theory / D. Sitnikov, O. Titova, S. Minukhin, A. Kovalenko, S. Titov // 2018 International Scientific-Practical Conference on Problems of Infocommunications Science and Technology, PICS\&T 2018 - Proceedings. - 2018. - P. 595-598.

12. Sitnikov, D. A method for association rule quality evaluation based on information theory / D. Sitnikov, E. Titova, O. Ryabov // WIT Transactions on Information and Communication Technologies. - 2006. - 37. - P. 25-34.

\section{References}

1. Agrawal, R. and Srikant, R. (1994), Fast algorithms for mining association rules, Proceedings of the $20^{\text {th }}$ VLDB Conference Santiago, Chile, pp. 487-499.

2. Agrawal, R. and Srikant, R. (1995), Mining generalized association rules, Proceedings of the 21th VLDB Conference Zurich, Swizerland, pp. 407-419.

3. WizWhy user's guide. Available at: https://www.wizsoft.com/n/downloads/User_Manual_WizWhy_4.pdf.

4. Chunhua Ju, Fuguang Bao, Chonghuan Xu and Xiaokang Fu (2015), A Novel Method of Interestingness Measures for Association Rules Mining Based on Profit, Hindawi Publishing Corporation Discrete Dynamics in Nature and Society, Vol. 2015. http://dx.doi.org/10.1155/2015/868634.

5. Victor, S.P. and Jeba, J.R. (2012), Effective measures in Association Rule Mining, International Journal of Scientific \& Engineering Research, Vol. 3, Issue 8.

6. Shaharanee, Izwan Nizal Mohd., Hadzic, Fedja and Dillon, Tharam S. (2011), Interestingness measures for association rules based on statistical validity, KnowledgeBased Systems, No.24, pp. 386-392. https://doi.org/10.1016/j.knosys.2010.11.005.

7. Prajapati, Dinesh J., Garg, Sanjay and Chauha, N.C. (2017), Interesting association rule mining with consistent and inconsistent rule detection from big sales data in distributed environment, Future Computing and Informatics Journal, No. 2, pp. 19-30.

8. Stephane, L., Teytaud, O. and Prudhomme, E. (2007), Association rule interestingness: measure and statistical valida- 
tion, Studies in Computational Intelligence (SCI), Vol. 43, pp. 251-275.

9. Aydin, T. and Guvenir, HA. (2009), Modeling interestingness of streaming association rules as a benefit-maximizing classification problem, Knowledge-based systems, Elsevier, pp. 85-99.

10. Sitnikov, D. and Titova, E. (2004), "Polnaya vzaimnaya informatsiya kak obobshchennyy pokazatel' kachestva assotsiativnykh zavisimostey s binarnymi priznakami" [Complete mutual information as a generalized indicator of the quality of associative dependencies with binary features], Information Processing Systems, No. 2, pp. 20-28.

11. Sitnikov, D., Titova, O., Minukhin, S., Kovalenko, A. and Titov, S. (2018), Informativity of Association Rules from the Viewpoint of Information Theory, Proceedings of International Scientific-Practical Conference on Problems of Infocommunications Science and Technology, (PICS\&T 2018), Kharkiv, pp. 595-598.

12. Sitnikov, D., Titova E. and Ryabov, O. (2006), A method for association rule quality evaluation based on information theory, WIT Transactions on Information and Communication Technologies, No. 37, pp. 25-34.

\section{Відомості про авторів:}

Ситніков Дмитро Едуардович

кандидат технічних наук

професор кафедри Харківського

національного університету радіоелектроніки,

Харків, Україна

https://orcid.org/0000-0003-1240-7900

\section{Ситнікова Поліна Едуардівна}

кандидат технічних наук

доцент кафедри Харківського

національного університету радіоелектроніки,

Харків, Україна

https://orcid.org/0000-0002-6688-4641

Тітов Сергій Володимирович

кандидат технічних наук

доцент кафедри Харківського

національного університету радіоелектроніки,

Харків, Україна

https://orcid.org/0000-0003-0910-4415

Тітова Олена Вітольдіївна

кандидат технічних наук

доцент кафедри Харківського

національного університету радіоелектроніки,

Харків, Україна

https://orcid.org/0000-0001-8894-2040

\section{Information about the authors:}

\section{Dmitro Sitnikov}

Candidate of Technical Sciences

Professor of Department of Kharkiv National

University of Radio Electronics,

Kharkiv, Ukraine

https://orcid.org/0000-0003-1240-7900

\author{
Polina Sitnikova \\ Candidate of Technical Sciences \\ Senior Lecturer of Kharkiv National \\ University of Radio Electronics, \\ Kharkiv, Ukraine \\ https://orcid.org/0000-0002-6688-4641
}

\author{
Serhii Titov \\ Candidate of Technical Sciences \\ Senior Lecturer of Kharkiv National \\ University of Radio Electronics, \\ Kharkiv, Ukraine \\ https://orcid.org/0000-0003-0910-4415
}

\author{
Olena Titova \\ Candidate of Technical Sciences \\ Senior Lecturer of Kharkiv National \\ University of Radio Electronics, \\ Kharkiv, Ukraine \\ https://orcid.org/0000-0001-8894-2040
}

\title{
ФИЛЬТРАЦИЯ РЕЗУЛЬТИРУЮЩЕГО НАБОРА АССОЦИАТИВНЫХ ПРАВИЛ С ТОЧКИ ЗРЕНИЯ ОЦЕНКИ ИНТЕРЕСНОСТИ
}

\author{
Д.Э. Ситников, П.Э. Ситникова, С.В. Титов, Е.В. Титова
}

Предлагается метод фильтрачии набора ассочиативных правил, полученных в результате поиска логических зависимостей. Количество найденных ассочиативных правил при установленных уровнях поддержки и доверия может быть достаточно большим и требует сокращения. Метод позволяет работать с так называемыми "интересными" правилами, которые имеют уровни поддержки и доверия, значительно отличающиеся от ожидаемых. Ожидаемые параметры рассчитываются исходя из предположения о независимости признаков, входящих в левую часть правила. Показано, как меняются уровни поддержски и доверия "интересных" ассочиативных правил при условии зависимости признаков в анализируемых данных.

Ключевые слова: ассоциативные правила, меры оценки ассоциативной зависимости, поддержка, доверие, уровень улучшения, интересные ассочиативные правила. 


\section{FILTRATION OF THE RESULTING SET OF ASSOCIATION RULES FROM THE POINT OF VIEW OF THE INTEREST ASSESSMENT}

D. Sitnikov, P. Sitnikova, S. Titov, O. Titova

One of the problems when generating association rules in Data Set is the fact that the number of dependencies can be quite large. Thus, the result set needs to filter the results in order to remove from it uninformative, duplicate rules. We propose a method for redacting a set of association rules obtained as a result of searching for logical dependencies in Data Set when Data Mining. The method allows working with so-called "interesting" rules, which have levels of support and confidence that differ significantly from the expected ones. Such an understanding of "interesting" association rules is offered, for example, by the developers of the WizWhy system, which allows you to work with the analyzed data table to find logical dependencies. The expected parameters are calculated based on the assumption of the independence of the attributes in the left part of the rule.

It is shown that if in the "interesting" rule the levels of support and confidence are lower than expected, then this indicates $a$ "negative" dependence of the attributes in the left part of the rule. Opposite, when the levels of support and confidence are higher than expected, we can talk about the "positive" dependence of the attributes in the left part of the logical dependence.

So in the case when the level of support (and as a consequence the level of confidence) is less than expected, the $A \rightarrow D$ and $B \rightarrow D$ rules should be left in the resulting set. Opposite, if the level of support and the level of trust is higher than expected, the $A \rightarrow D$ or $B \rightarrow D$ rule remains in the resulting set.

Also, this method allows you to calculate the parameters of the dependency (level of support and confidence) between the attributes in the left side of the "interesting" rule. Thus, provided that "interesting" rules are found, the resulting set can be supplemented by an associative rule that characterizes the relationship between the attributes on the left of the association.

The proposed method allows you to filter "interesting" association rules that have only two attributes on the left. In what follows we will consider "interesting" rules with a large number of attributes.

Keywords: association rules, measures for assessing associative dependence, support, confidence, improvement, interesting association rules. 